\title{
Gewinnung von Morin und Moringerbsäure (= Maclurin);
}

nach Hlasiwetz und Pfaundler.

Das geraspelte Gelbholz (von Maclura tinctoria) wird 2 bis 3 Mal mit Wasser ausgekocht, die abgeseihte Flüssigkeit auf die Hälfte des Gewichts des angewandten Holzes eingedampft und mehre Tage stehen gelassen. Man findet dann eine ziemliche Menge eines krystallinischen gelben Absatzes, der aus Morin, Morinkalk und Moringerbsäure besteht; aus den Mutterlaugen bildet sicb nach längerem Stehen noch mehr desselben. Man sammelt ihn auf Leinen, spült ihn mit Isaltem Wasser etwas ab, kocht ihn 2 Mal nach einander mit Wasser aus und filtrirt die Abkochungen siedend. Hierbei löst sich Moringerbsäure, während Morin und Morinkalk ungelöst bleiben. Dieses letztere Gemenge erhitzt man mit salzsäurehaltigem Wasser, um den Kalk auszuziehen, wäscht das Ungelöste mit Wasser gut aus, löst es in heissem Weingeist, filtrirt heiss und mischt das Filtrat sogleich mit $2{ }_{3}$ seines Volumens heissem Wasser. In der Ruhe krystallisirt daraus das Morin in gelben Nadeln fast vollständig heraus; durch mehrmaliges Umkrystallisiren aus schwachem Weingeist wird es gereinigt. Die Lösungen müssen durch $\mathrm{CaO}$. und $\mathrm{Fe}^{2} \mathrm{O}^{3}$ freie Filter filtrirt werden. Zur Entfernung bräunlicher Zersetzungsproducte bewirkt man in der Morinlösung durch Bleizucker einen Niederschlag, zersetzt denselben in der erwärmten Flüssigkeit durch HS und filtrirt die nun noch strohgelbe Lösung des reinen Morins vom PbS ab. Fallt man diese Lösung dann mit Wasser, so erscheint der Morinniederschlag fast farblos.

Eigenschaften des Morins $=\mathrm{C}^{24} \mathrm{H}^{8} \mathrm{O}^{10}+3 \mathrm{HO}$

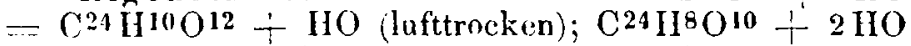
(bei $100^{0} \mathrm{C}$. getrocknet); $\mathrm{C}^{24} 1^{8} \mathrm{O}^{10}+\mathrm{HO}$ (bei $120^{\circ}$ bis $2000($ ) $) 2\left(\mathrm{C}^{24} \mathrm{H}^{8} \mathrm{O}^{10}\right)+\mathrm{HO}$ (bei 250 bis $2980 \mathrm{C}$. getrocknet). Das Morinanhydrid $=\mathrm{C}^{24} \mathrm{H}^{8} \mathrm{O}^{10}$ sublimirt unzersetzt, obgleich schwierig nahe bei $300^{\circ} \mathrm{C}$. in gelblichen Krystallen.

Das lufttrockene Morin bildet glïnzende zu Büscheln verwachsene, 1 bis 3 Iinien lange gelbe Nadeln. Es ist fast unlöslich in kaltem, setır wenig löslich in siedenden Wasser, leicht löslich in Alkohol, weniger leicht löslich in Aether, unlöslich in $\mathrm{C}^{2} \mathrm{~S}^{4}$, löslich in Essigsäure. Alkalien und alkalisch reagirende Salze wie phosphorsaures und borsaures Natron lösen es sehr schnell mit tiefgelber 
Farbe; Säuren fällen daraus unverändertes Morin. Eisenchlorid färbt die alkoholische Lösung tief olivengrün.

Eine ammoniakalische Lösung des Morins reducirt aus $\mathrm{AgO}, \mathrm{NO}^{5}$ schon in der Kälte metallisches silber. Noch eine sehr verdünnte alkalische Morinlösung reducirt das $\mathrm{Cu}^{2} \mathrm{O}^{2}$ zu Cu $\mathrm{Cu}^{2} \mathrm{O}$ bei der Trommer'schen Probe. Das lufttrockene Morin verliert bei Temperaturen bis $298^{\circ} \mathrm{C}$. erhalten höchstens 10 Proc. HO. Die Schwierigkeit das HO völlig und ohne die Substanz zu zersetzen auszutreiben, theilt das Morin mit dem Quercetin.

Morinkali $=\mathrm{KO}, \mathrm{C}^{24} \mathrm{H}^{9} \mathrm{O} 11$, gelbe leichte Nadeln.

Morinnatron $=\mathrm{NaO}, \mathrm{C}^{24} \mathrm{H}^{9} \mathrm{O}^{11}$, desgleichen.

Morinkalk $=\mathrm{CaO}, \mathrm{C}^{24} \mathrm{H}^{9} \mathrm{O}^{11}$, gelber Niederschlag durch $\mathrm{CaCl}$ in Morinkalilösung.

Morinbaryt $=\mathrm{BaO}, \mathrm{C}^{24} \mathrm{H}^{9} \mathrm{O}^{11}$.

Morinbleioxyd, gelber Niederschlag, den alkoholische Bleizuckerlösung in alkoholischer Morinlösung bewirkt, mit 44,3 bis 45,8 Proc. Bleioxydgehalt.

Morinzinkoxyd $=\mathrm{ZnO}, \mathrm{C}^{24} \mathrm{H}^{9} \mathrm{O}^{11}$, seideglänzende Nadeln, unlöslich in Wasser.

Morinammoniak $=2 \mathrm{H}^{3} \mathrm{~N}, \mathrm{C}^{24} \mathrm{H}^{8} \mathrm{O}^{10}$, intensiv gelbe Verbindung.

Brommorin $=\mathrm{C}^{24} \mathrm{H}^{7} \mathrm{Br}^{3} \mathrm{O}^{12}$ nadelförmige Krystalle.

Löst man Morin in Wasser auf, dem man einige Tropfen Natronlauge zugesetzt hat und bringt Natriumamalgam hinzu, so bemerkt man indigblaue Färbung, darauf wird das Gemisch grün, dann gelbbraun, zuletzt strohgelb. Jetzt bewirkt' Salzsäure keine Abscheidung von Morin mehr, denn dieses ist vollständig in Phloroglycin $\mathrm{C}^{12} \mathrm{H}^{6} \mathrm{O}^{6}$ verwandelt, welches man der sauren Lösung durch Schütteln mittelst Aether entziehen kann. $\mathrm{C}^{24} \mathrm{H}^{10} \mathrm{O}^{12}+2 \mathrm{H}=2 \mathrm{C}^{12} \mathrm{H}^{6} \mathrm{O}^{6}$.

Wirkt Natriumamalgam auf eine mit $\mathrm{HCl}$ angesäuerte Morinlösung, so nimmt die Flüssigkeit, die man fortwährend durch $\mathrm{HCl}$ sauer erhält, eine Purpurfarbe an, wird dann rothgelb, rein gelb, zuletzt gelblich und entbält dann ebenfalls Phloroglycin.

Unterbricht man aber die Einwirkung des Natriumamalgams, sobald die Purpurfarbe am intensiveten ist, indem man die Flüssigkeit vom Amalgam abgiesst, vom abgeschiedenen $\mathrm{NaCl}$ abfiltrirt und im Wasserbade concentrirt, so erhält'man glänzend purpurrothe Prismen von Isomorin $\mathrm{C}^{24} \mathrm{H}^{8} \mathrm{O}^{10}+2 \mathrm{HO}$, deren alkoholische Lösung einen Dichroismus wie Uranglas zeigt (gelbe Farbe und grünen Reflex); mit Alkalien wird sie 
grasgrün. Beim Erwärmen der alkalischen Lösung, bei längerem Stehen derselben wird sie durch Bildung von gewöhnlichem Morin wieder weingelb. Isomorin bildet sich auch bei Behandlung einer Lösung von Morin in erwärmter concentrirter $\mathrm{HO}, \mathrm{SO}^{3}$ und Behandlung der mit etwas Wasser verdünnten Lösung mit granulirtem Zink; das Endproduct dieser Reaction ist aber auch hier Phloroglycin.

Morin mit Kalihydrat geschmolzen liefert Phloro-

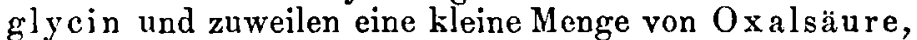
somit kein weiteres Zersetzungsproduct. Fs sind bekanntlich vornehmlich Verbindungen von der Natur der Aldehyde, die sowohl mit $\mathrm{H}$, als auch mit $\mathrm{KO}, \mathrm{HO}$ identische Producte liefern können, z. B. Bittermandelöl, welches in beiden Fällen Benzalkobol liefert.

Unter den Producten der trockenen Destillation des Morins findet sich etwas Phloroglycin.

\section{M acI urin (sogenannte Moringerbsäure).}

Dasselbe befindet sich in der vom rohen Morin ab. filtrirten Flüssigkeit. Man dampft sie etwas ein und erhält nach dem Erkalten einen Theil desselben in Krystallen, aus der davon abfiltrirten Mutterlauge fällt auf Zusatz von $\mathrm{HCl}$ eine neue Menge desselben. Man reinigt durch wiederholtes Umkrystallisiren aus salzsaurem Wasser ( $\mathrm{HCl}$ zersetzt das Maclurin nicht). Nach Delffs entfärbt man die Krystalle durch Pressen zwischen Papier unmittelbar nach dem Anschiessen. Zuletzt erhält man farblose Krystalle. Auch durch Aufösen der Krystalle in heissem Wasser, Zusatz von etwas Essigsäure und Bleizucker, Einleiten von HS entfärbt das $\mathrm{PbS}$ die Lösung bis auf schwach gelbliche Färbung und man erhält eben solche Krystalle. Die Trennung des Maclurins von Morin beruht also auf der reichlichen Löslichkeit des Maclurins in heissem Wasser, worin sich Morin nur spurenweise löst.

Das Maclurin wird erst bei 130 bis $140^{\circ} \mathrm{C}$. wasserfrei $=\mathrm{C}^{26} \mathrm{H}^{10} \mathrm{O}^{12}$. Das bei $1000 \mathrm{C}$. getrocknete enthält noch $2 \mathrm{HO}$ bis $3 \mathrm{HO}$.

Behandelt man dasselbe mit concentrirter Lösung von Aetzkali in der Hitze, so zerfällt es in Phloroglycin und Protocatechusäure nach der Gleichung

$\mathrm{C}^{26} \mathrm{H}^{10} \mathrm{O}^{12}+2 \mathrm{HO}=\mathrm{C}^{12} \mathrm{I1}^{6} \mathrm{O}^{6}+\mathrm{C}^{24} \mathrm{H}^{6} \mathrm{O}^{8}$.

Das Maclurin kann also als protocatechusaures Phloroglycin $=$ Phloroglycin-Protocatechusäure $\mathrm{C}^{12} \mathrm{H}^{5} \mathrm{O}^{5}, \mathrm{C}^{14} \mathrm{H}^{5} \mathrm{O}^{7}$ angesehen werden. 
Der Name Moringerbsäure für diese Verbindung ist unpassend, da dieselbe keine Säure ist.

Kocht man eine nicht zu concentrirte Lösung von Maclurin mit Zink und verdünnter Schwefelsäure, so liefert es $\mathrm{Phloroglycin} \mathrm{C}^{12} \mathrm{H}^{6} \mathrm{O}^{6}$ und $\mathrm{Machromin}=$ $\mathrm{C}^{28} \mathrm{H}^{10} \mathrm{O} 10$. Letzteres entsteht dabei aus der Protocatechusiiure nach der Gleichung

$2 \mathrm{C}^{14} \mathrm{H}^{6} \mathrm{O}^{8}+4 \mathrm{H}=\mathrm{C}^{28} \mathrm{H}^{10} \mathrm{O}^{10}+6 \mathrm{HO}$.

Das Machromin $\mathrm{C}^{28} \mathrm{H}^{10} \mathrm{O}^{10}+6 \mathrm{HO}$ bildet farblose flimmernde Kryställchen (feine Nädelchen), die sich an der Luft und im Lichte, so wie beim Trocknen in der Wärme rasch dunkelblau färben. Schwerlöslich selbst in heissem Wasser, leicht löslich in Alkohol und Aether. Eine wässerige kochend bereitete Lösung wird allmälig beim Stehen an der Luft tief veilchenblau und giebt auf $\mathrm{HCl}$-Zusatz einen amorphen indigblauen Niederschlag.

Eisenchlorid giebt in einer sehr verdünten weingeistigen Lösung des Machromins eine schön violettrothe, weiterhin königsblau werdende Färbung.

Trommer'sche Kupferoxydlösung wird durch Machromin beim Erwärmen reducirt.

Concentrirte $\mathrm{HO}, \mathrm{SO}^{3}$ löst das Mochromin mit or angerother, dann gelb, beim Erwärmen smaragdgrün werdender Farbe; diese bleibt auch beim Verdünnen, mit Alkali übersättigt, wird die Flüssigkeit violett. Die $6 \mathrm{HO}$ entweichen bei 100 bis $130^{\circ} \mathrm{C}$. Machromin wird durch Bleizucker gefällt.

Der aus Maclurin durch Eisenchlorid erzeugte dunkelindigblaue Körper löst sich leicht in Alkohol und wird daraus durch Wasser nicht gefallt.

Die angesäuerte Lösung wird durch $\mathrm{Zn}$ - oder Naamalgam entfärbt und giebt dann an Aether wieder $\mathrm{Ma}$ chromin ab. Das Machrominblau hat die Formel $\mathrm{C}^{28} \mathrm{H}^{8} \mathrm{O}^{10}$.

Bei Einwirkung von Natriumamalgam auf Maclurin in alkalischer Lösung entstehen Phloroglycin und ein amorpher in Wasser, Alkohol und Aether löslicher, durch Bleizucker fällbarer Körper $=\mathrm{C}^{28} \mathrm{H}^{12} \mathrm{O}^{10}$, der mit Eisenchlorid eine grusgrüne Färbung giebt, die mit $\mathrm{NaO}, \mathrm{CO}^{2}$ roth wird; er giebt bei der trocknen Destillation Krystalle von Brenzcatechin.

$$
2 \mathrm{C}^{14} \mathrm{H}^{6} \mathrm{O}^{8}+6 \mathrm{H}=\mathrm{C}^{28} \mathrm{H}^{12} \mathrm{O}^{10}+6 \mathrm{HO} \text {. }
$$

Protocatechusäure $=\mathrm{C}^{14} \mathrm{H}^{6} \mathrm{O}^{8}$. Die nächsten Reactionen für ihre Erkennung sind die eigenthümlich blaugrüne Färbung ibrer Lösung mit Eisenoxydsalzen, das 
Rothwerden dieser Färbung auf Zusatz von $\mathrm{NaO}, \mathrm{CO}^{2}$ und die schön violette Färbung, welche die Săurelösung mit Eisenoxydsalzen giebt, wenn man sie zuvor mit $\mathrm{BaO}^{2} \mathrm{CO}^{2}$ abgesättigt hat. (Diese Reaction theilt sie mit dem Brenzcatechin. Hlasiwetz.) (Wien. Sitzungsber. der k. k. Akad. der Wiss. Math.-phys. Cl. 50. Bd. 1. Heft. II. Abth. S.6-57. Vergl. auch Ann. d. Chem. u. Phar'm. Bd. 127. S. 351 und Arch. d. Pharm. Bd. 113. S. 255.)

H. Ludwig.

\section{Quercitrin.}

Hlasiwetz und $P$ faundler geben demselben die Formel $\mathrm{C}^{66} \mathrm{H}^{30} \mathrm{O}^{34}$ (wasserfrei). Die Spaltung desselben in Quercetin $=\mathrm{C}^{54} \mathrm{H}^{18} \mathrm{O}^{24}$ und in Isodulcit $=$ $\mathrm{C}^{12} \mathrm{H}^{14} \mathrm{O}^{12}$ wird ausgedrückt durch

$\mathrm{C}^{66} \mathrm{H}^{30} \mathrm{O}^{34}+2 \mathrm{HO}=\mathrm{C}^{54} \mathrm{H}^{18} \mathrm{O}^{24}+\mathrm{C}^{12} \mathrm{H}^{14} \mathrm{O}^{12}$. Wasserhaltiges Quercitrin $=\mathrm{C}^{66} \mathrm{H}^{30} \mathrm{O}^{34}+2 \mathrm{HO}$ oder $+4 \mathrm{HO}$ oder $+6 \mathrm{HO}$.

Quercetin $=\mathrm{C}^{54} \mathrm{H}^{18} \mathrm{O}^{24} \mathrm{kann}$ durch die Formel repräsentirt werden

$$
\begin{aligned}
& \mathrm{C}^{24} \mathrm{H}^{8} \mathrm{O}^{10}+\mathrm{C}^{30} \mathrm{H}^{10} \mathrm{O}^{14}=\mathrm{C}^{54} \mathrm{H}^{18} \mathrm{O}^{24} \\
& \text { Morin } t \text {-Quercetinsäure }=\text { Quercetin }
\end{aligned}
$$

Quercetinsäure $=\mathrm{C}^{30} \mathrm{H}^{10} \mathrm{O}^{14} \mathrm{kann}$ zerfallen in $\mathrm{C}^{16} \mathrm{H}^{6} \mathrm{O}^{10}=\mathrm{Quercimerins} a$ ure und in $\mathrm{C}^{14} \mathrm{H}^{6} \mathrm{O}^{8}=$ Protocatechusäure.

Quercimerins äure $+20=$ Protocatechusäure $+\mathrm{C}^{2} \mathrm{O}^{4}$.

Quercetin liefert mit Kalihydrat geschmolzen $\mathrm{Phlo-}$ rogly cin $=\mathrm{C}^{12} \mathrm{H}^{6} \mathrm{O}^{6}$. (Wien. Sitzungsber. der k.k. Akad. der Wissensch. I1. Abth. S. 52. Vergl. Arch. d. Pharm. Bd. 113. S. 256.)

H. Ludwig.

\section{Ueber Catechu, Catechin und Kino}

hat H. Hlasiwetz Mittheilungen gemacht. Rohes Catechu sowohl wie auch das daraus dargestellte Catechin liefern, wenn man sie in dem Verhältnisse von $1: 3$ mit Kalihydrat schmilzt, Protocatechusäure $=\mathrm{C}^{14} \mathrm{H}^{6} \mathrm{O}^{8}$ und Phloroglycin $=\mathrm{C}^{12} \mathrm{H}^{6} \mathrm{O}^{6}$, wie J. Malin durch Versuche zeigte. Kraut und van Delden fanden schon die I'rotocatechusäure, aber nicht das Phloroglycin. Sie stellen für das Catechin die Formel $\mathrm{C}^{24} \mathrm{H}^{12} \mathrm{O}^{10}$ auf und sagen: „Das Catechin enthält den Atomencomplex des Brenzcatechins zweimal oder, was wahrscheinlicher ist, 